\title{
Visual emotional information processing in male schizophrenia patients: Combining ERP, clinical and behavioral evidence
}

\author{
Ana P. Pinheiro ${ }^{\mathrm{a}, \mathrm{b}}$, Taosheng Liu ${ }^{\mathrm{c}}$, Paul G. Nestor ${ }^{\mathrm{b}, \mathrm{d}}$, Robert W. McCarley ${ }^{\mathrm{b}}$, \\ Óscar F. Gonçalves ${ }^{\mathrm{a}}$, Margaret A. Niznikiewicz ${ }^{\mathrm{b}, *}$ \\ a Neuropsychophysiology Lab, CIPsi, School of Psychology, University of Minho, Braga, Portugal \\ b Laboratory of Neuroscience, Department of Psychiatry, Clinical Neuroscience Division, Boston VA Healthcare System, Brockton Division and Harvard \\ Medical School, 940 Belmont Street, Brockton, MA, United States \\ ${ }^{\mathrm{c}}$ Department of Psychology, Second Military Medical University (SMMU), Shanghai, China \\ d University of Massachusetts, Boston, MA, United States
}

\section{A R T I C L E I N F O}

\section{Article history:}

Received 17 March 2013

Received in revised form 30 May 2013

Accepted 14 June 2013

\section{Keywords:}

Schizophrenia

Affective processing

Mood

IAPS pictures

Event-related potentials

\begin{abstract}
A B S T R A C T
We used event-related potentials (ERPs) to examine the time course of visual emotion processing in schizophrenia. ERPs were recorded in 19 chronic male schizophrenia patients and 16 healthy controls (HC) while they viewed IAPS pictures used to induce positive, negative, or neutral mood, presented in a blocked design across three separate occasions. Electrophysiologically, groups differences were found in early potentials (the first $200 \mathrm{~ms}$ of picture viewing): C1, P1, and N1; also, less positive, broadly distributed P2 was found in the patient group. P2 amplitude to negative valenced pictures only was correlated with self-reported negative mood in the patients. The groups did not differ in late-occurring potentials, specifically the late positive potential (500-1000 ms latency window). Patients reported more negative affect before and after the three types of mood induction; however, mood induction influenced both groups in the same direction. Both groups showed similar subjective valence and arousal ratings of evocative stimuli. The ERP results suggest that visual evocative stimuli are differentially processed within the first $200 \mathrm{~ms}$, and that the early stages of visual evocative stimuli processing are abnormal in schizophrenia, irrespective of stimulus valence. The correlation found between sensory abnormalities in negative pictures processing and negative mood suggests a relationship between abnormal sensory processes and increased negative mood experience in patients.
\end{abstract}

Published by Elsevier Ireland Ltd.

\section{Introduction}

Abnormalities in emotion processing are a hallmark of schizophrenia, and have been associated with impaired social interactions and functional outcome in this disorder [4]. Abnormalities include impairments in emotional perception [5] and in emotional expression [14] that coexist with less impaired subjective experience of emotion [17].

Investigations of how affective stimuli are processed by schizophrenia patients and how they affect the way patients feel has mostly used visual evocative stimuli such as pictures or facial expressions [11]. Behavioral studies of visual emotional perception have demonstrated impaired recognition of emotion from visual cues [5]. In addition, the few published event-related

\footnotetext{
* Corresponding author at: Psychiatry 116A, Boston VA Healthcare System, 940 Belmont Street, Brockton, MA 02301, United States. Tel.: +1 774826 1507; fax: +1 7748261299 .

E-mail address: Margaret_Niznikiewicz@hms.harvard.edu (M.A. Niznikiewicz).
}

potential (ERP) studies pointed to abnormalities in either early and/or late stages of visual emotional processing, depending on the experimental task $[16,17]$. These studies used pictures from the International Affective Pictures System (IAPS [19]) in taskrelevant [17] and task-irrelevant [16] conditions. In a passive picture viewing task [17], subjects saw pleasant, unpleasant and neutral pictures presented each for $6 s$ and repeated during the experiment. Schizophrenia patients rated the valence of IAPS pictures similarly to healthy controls but differed in the late positive potential (LPP), with patients exhibiting no significant difference between LPP amplitude for pleasant and neutral stimuli. These results were interpreted as evidence for preserved initial sensory processing of emotional visual information and resource allocation to emotional stimuli in schizophrenia, coexisting with disrupted sustained attentional processing of emotional pictures. In a taskirrelevant task, neutral, pleasant and unpleasant IAPS pictures were presented in a modified visual $\mathrm{P} 300$ paradigm involving target letter detection [16]. No group differences were observed in the early posterior negativity (EPN) and LPP. These results replicated preserved automatic capture of attention by affective stimuli found in 
the Horan et al. [17] study and suggested that sustained processing of affective stimuli is also intact when stimuli are task-irrelevant and presented outside of a focus of attention. These results also suggested that the type of task and methodological details of design have an important effect on the pattern of results obtained. For example, in addition to the different tasks used in both Horan et al. $[16,17]$ studies, IAPS pictures with different valence were presented in the same session (mixed design). It is thus possible that the processing of a given picture was 'contaminated' by the effects elicited by a previously presented picture. Also, the stimuli repetition in the Horan et al. $[16,17]$ studies may have resulted in the ERP repetition effects that might have contributed to the pattern of observed results.

In the studies focusing specifically on abnormalities in visual processing, dysfunction in early stage visual processing was associated with deficits in higher-level cognitive functioning in schizophrenia, such as the assignment of meaning to a salient visual stimulus [5,6]. A relationship was also found between abnormal visual processing and measures of social perception in schizophrenia [24].

The investigation of emotional experience in schizophrenia sheds light on how evocative stimuli impact patients' mood or feeling states. These studies have also reported conflicting results. Some studies found similar levels of mood change in schizophrenia individuals compared with $\mathrm{HC}$, while others reported a reduced response to mood induction in patients, particularly for positive facial expressions [23]. The studies examining knowledge representations of visual affective stimuli in schizophrenia reported similar assessment of stimuli's valence (i.e., the overall unpleasantness/displeasure relative to pleasantness/attraction of a stimulus) and arousal (i.e., the intensity of motivational mobilization - appetitive or defensive) [17]. However, in other studies, schizophrenia individuals rated pleasant stimuli as less pleasant and negative stimuli as less unpleasant [8]. Differences in patients' samples (e.g., schizophrenia subtype, clinical symptoms and functional outcome measures), and in experimental stimuli (e.g., level of arousal) may account for these discrepancies.

Given the conflicting results between task-relevant and task irrelevant designs, as well as evidence of early visual abnormalities in schizophrenia, this study investigated the temporal course of evocative pictures processing in schizophrenia in the context of a mood induction procedure, using ERP, clinical and behavioral measures. In addition, in contrast to the previous studies [16,17] where all valence types of stimuli were presented in the same block, we presented each valence type in a separate block, with no stimulus repetition (blocked design). We analyzed early sensory visual potentials, mid-latency and late components.

The hypotheses addressed contrasting effects reported in studies using task-irrelevant and task relevant designs:

1) Since in task-irrelevant designs the processing of affective stimuli was found normal at both early $[16,17]$ and late processing stages [16], we predicted no differences in the early, mid-latency and late ERP components.

2) Given the reports of abnormal visual sensory processing of affective stimuli [9], and abnormal late processing of affective stimuli in task-relevant designs [17], we predicted abnormalities in early and late ERP components.

Abnormalities in early visual ERP components for both neutral and emotional pictures would indicate a more generalized visual impairment in schizophrenia. An impairment in the processing of emotional vs. neutral visual information indexed by early visual ERP components would suggest that emotion impacts differentially early visual processing abnormality.
We additionally tested the association between early visual abnormalities and emotional processing in schizophrenia.

\section{Materials and methods}

\subsection{Participants}

Nineteen male subjects diagnosed with chronic schizophrenia and sixteen male HC, participated in the study (see Table 1). Inclusion criteria were: age 18-50 years; right-handedness [21]; American English as first language; normal or corrected-to-normal vision; no history of neurological illness or a traumatic head injury; no history of alcohol or drug dependence in the past five years or abuse within the last year (DSM-IV-TR) with diagnoses determined by the Structured Clinical Interview for DSM-IV (SCID $[9,10])$; the use, in the preceding year, of any drug or medication affecting electroencephalogram (EEG) morphology, or with consequences for neurological and/or cognitive functioning such as steroids or barbiturates; no hearing, vision or upper body impairment; estimated intelligence quotient (IQ) of above 80 [25]; no alcohol use in the $24 \mathrm{~h}$ before testing. All participants signed a written informed consent, following Harvard Medical School and Veterans Affairs Boston Healthcare System guidelines.

Comparison subjects were recruited from Internet advertisements, and matched to the patients on the basis of age, handedness, gender and parental socio-economic status [15]. For HC, additional inclusion criteria were: no history of Axis I [10] or II [9]; no history of Axis I disorder in first or second degree family members.

Clinical measures included the SCID-I and II $[9,10]$, the Positive and Negative Syndrome Scale (PANNS [18]), the Scale for the Assessment of Positive Symptoms (SAPS [2]), and the Scale for the Assessment of Negative Symptoms (SANS [1]). Participants also completed the POMS questionnaire [20] before and after they viewed the pictures in order to assess the mood at the study entry and the effectiveness of the mood induction procedure.

\subsection{Stimuli}

Stimuli were 30 neutral, 30 positive and 30 negative valence pictures selected from the IAPS database [19]. ${ }^{1}$ Images were matched on overall content: they contained human figures, animals, scenery, objects, and real-world settings. Highly arousing pictures involving violence, physical injuries/deformities, or nudity were excluded. IAPS norms for the American population for the items used in the study both in terms of valence (1-9 corresponding to unpleasantto-pleasant range) and arousal (low-to-high) are presented in Table 2. Valence $(F(2,89)=2640.16, p<0.001)$ and arousal $(F(2$, $89)=336.84, p<0.001)$ differed across the three categories. Stimuli were presented and synchronized through Superlab 4.2.

\subsection{Procedure}

Participants were tested in three sessions (one session for each emotional valence) occurring on different days, conducted in a soundproof, electrically-shielded chamber. They were seated in a comfortable chair 40 inches in front of a 17" CRT monitor. Each picture was presented for $1000 \mathrm{~ms}$, and preceded by a fixation

\footnotetext{
1 IAPS pictures included: Negative - 2141, 2276, 2278, 2375, 2455, 2700, 2750 2799, 2900, 3181, 3300, 6311, 6561, 9000, 9007, 9041, 9102, 9180, 9265, 9290 , 9320, 9419, 9421, 9432, 9435, 9452, 9471, 9561, 9584, 9830; Positive - 1440, 1441 1460, 1610, 1750, 1811, 1920, 2040, 2050, 2057, 2070, 2080, 2150, 2154, 2160, 2165 , $2170,2260,2311,2332,2340,2530,2540,2550,2660,5600,5780,5830,5831,7580$ Neutral - 2038, 2880, 5130, 5510, 5520, 5530, 6150, 7000, 7002, 7004, 7006, 7010 7020, 7034, 7035, 7041, 7050, 7053, 7055, 7056, 7059, 7080, 7090, 7179, 7187, 7217. 7233, 7235, 7490, 7950.
} 
Table 1

Demographic and clinical characteristics of participants.

\begin{tabular}{|c|c|c|c|}
\hline Variable & Healthy controls $(n=16)$ & Schizophrenia patients $(n=19)$ & $p$ Value \\
\hline Age (years) & $46.2 \pm 7.19$ & $46.9 \pm 9.41$ & .793 \\
\hline Education (years) & $15.3 \pm 1.80$ & $13.3 \pm 2.07$ & $.005^{*}$ \\
\hline Subject's SES ${ }^{a}$ & $2.0 \pm 0.89$ & $3.4 \pm 1.30$ & $.001^{*}$ \\
\hline Parental SES & $2.4 \pm 1.09$ & $2.8 \pm 1.26$ & .253 \\
\hline Handedness $^{\mathrm{b}}$ & $0.72 \pm 0.28$ & $0.77 \pm 0.22$ & .591 \\
\hline Onset age (years) & NA & $27.2 \pm 8.67$ & NA \\
\hline Duration (years) & NA & $13.9 \pm 9.23$ & NA \\
\hline Chlorpromazine EQ (mg) & NA & $508.73 \pm 447.88$ & NA \\
\hline Antipsychotic medication type & NA & $\begin{array}{l}\text { Typical (fluphenazine decanoate, proloxin decanoate, haloperidol)=1; } \\
\text { Atypical (risperidone, olanzapine, ziprasidone, quetiapine, } \\
\text { aripiprazole)=13; Both }=2\end{array}$ & NA \\
\hline Other psychotropic medication & NA & $\begin{array}{l}\text { Antidepressants (sertraline, citalopram, bupoprion, trazodone) }=4 \\
\text { Benzodiazepines (lorazepam, clonazepam) }=4 \\
\text { Lithium carbonate = } 2 \\
\text { Valproic acid = } 3\end{array}$ & NA \\
\hline PANSS positive scale & NA & $23.4 \pm 8.97$ & NA \\
\hline PANSS negative scale & NA & $21.1 \pm 9.46$ & NA \\
\hline PANSS general psychopathology & NA & $41.4 \pm 16.16$ & NA \\
\hline PANSS total psychopathology & NA & $85.8 \pm 30.95$ & NA \\
\hline SANS anhedonia & NA & $3.11 \pm 1.49$ & NA \\
\hline
\end{tabular}

Notes. SES = socioeconomic status; Chlorpromazine EQ= chlorpromazine equivalent; $\mathrm{NA}=$ not applicable.

a Hollingshead four-factor index of social status [15].

b Edinburgh Handedness Inventory [24].

* $p<0.01$.

cross $(1000 \mathrm{~ms})$. After the picture, a blank screen was presented for $2000 \mathrm{~ms}$, until the beginning of the next trial. The order of pictures' valence was counterbalanced across subjects. No picture was repeated in the three experimental sessions.

Subjects were told to view the pictures to help decide how well they were suited for a future mood experiment. They were asked to not make any responses during the EEG session. After the ERP experiment, in a separate session, participants viewed the pictures again to explicitly assess the valence and arousal of each picture (presented for $10 \mathrm{~s}$ ), with a computerized version of the SelfAssessment Manikin [3] where each affective dimension is assessed on a 1-9 Likert scale: higher numbers indicate evaluation as more pleasant or arousing.

\subsection{EEG recording}

EEG was recorded during IAPS pictures presentation using the ActiveTwo BioSemi system (BioSemi B.V., Amsterdam, Netherlands) and a 64-channel custom-designed electrode cap. EEG was acquired in a continuous mode, digitized at $512 \mathrm{~Hz}$, with a bandpass of DC- $100 \mathrm{~Hz}$, and stored for later analysis. Blinks and eye movements were monitored via electrodes placed on left and right temples and one above the left eye. The voltage at each electrode was measured online with respect to a common mode sense electrode.

\subsection{EEG data analysis}

Analysis of EEG data was conducted with Analyzer software package (Brain Products, Inc., 2000). Data were re-referenced off-line to the average of the left and right mastoids. Individual ERP epochs were constructed between -100 and $1000 \mathrm{~ms}$ poststimulus, with eye blink and movement artifacts corrected by the Gratton et al. [13] method. Trials containing muscle activity or amplifier blocking were rejected off-line before averaging. Artifact rejection was performed for any trial that exceeded $\pm 100 \mu \mathrm{V}$. Separate averages were calculated for each stimulus type (pleasant, unpleasant, and neutral), after subtraction of the $100 \mathrm{~ms}$ pre-stimulus baseline. The number of individual trials did not differ between groups $(p>0.05)$ : pleasant stimuli $-24 / 30$ in $\mathrm{HC}$ and 26/30 in patients; unpleasant stimuli $-25 / 30$ in HC and $25 / 30$ in patients; neutral stimuli - 26/30 in HC and 26/30 in patients.

Distinct positive and negative components were identified in two different scalp regions: early sensory components localized to posterior locations: $\mathrm{C} 1, \mathrm{P} 1$, and $\mathrm{N} 1$; anterior locations: $\mathrm{N} 1$; two broadly distributed components: P2 and LPP. The components' amplitudes were measured as mean activity in a specified latency window post-stimulus onset: (a) posterior components - C1: 50-110 ms; P1: 110-150 ms; N1: 150-210 ms; (b) anterior N1: 120-190 ms; (c) Broadly distributed components - P2: 200-270 ms; LPP: 500-1000 ms.

Table 2

Mean (SD) ratings of IAPS visual stimuli.

\begin{tabular}{|c|c|c|c|c|}
\hline & Condition & IAPS normative sample & Healthy control subjects & Schizophrenia patients \\
\hline \multirow[t]{3}{*}{ Valence $^{\mathrm{a}}$} & Neutral & $5.05(0.19)$ & $5.08(0.72)$ & $5.04(1.11)$ \\
\hline & Positive & $7.85(0.25)$ & 7.28 (1.09) & $7.28(0.70)$ \\
\hline & Negative & $2.74(0.35)$ & $2.49(0.77)$ & $2.80(0.73)$ \\
\hline \multirow[t]{3}{*}{ Arousal $^{\mathrm{b}}$} & Neutral & $2.65(0.36)$ & $2.78(1.59)$ & $3.63(1.38)$ \\
\hline & Positive & $4.48(0.38)$ & $5.12(2.57)$ & $5.53(1.91)$ \\
\hline & Negative & $4.78(0.28)$ & $4.70(2.60)$ & $6.26(1.72)$ \\
\hline
\end{tabular}

a Ratings on a 9-point scale, from 1 (totally unpleasant) to 9 (totally pleasant).

b Ratings on a 9-point scale, from 1 (totally calm) to 9 (totally aroused). 

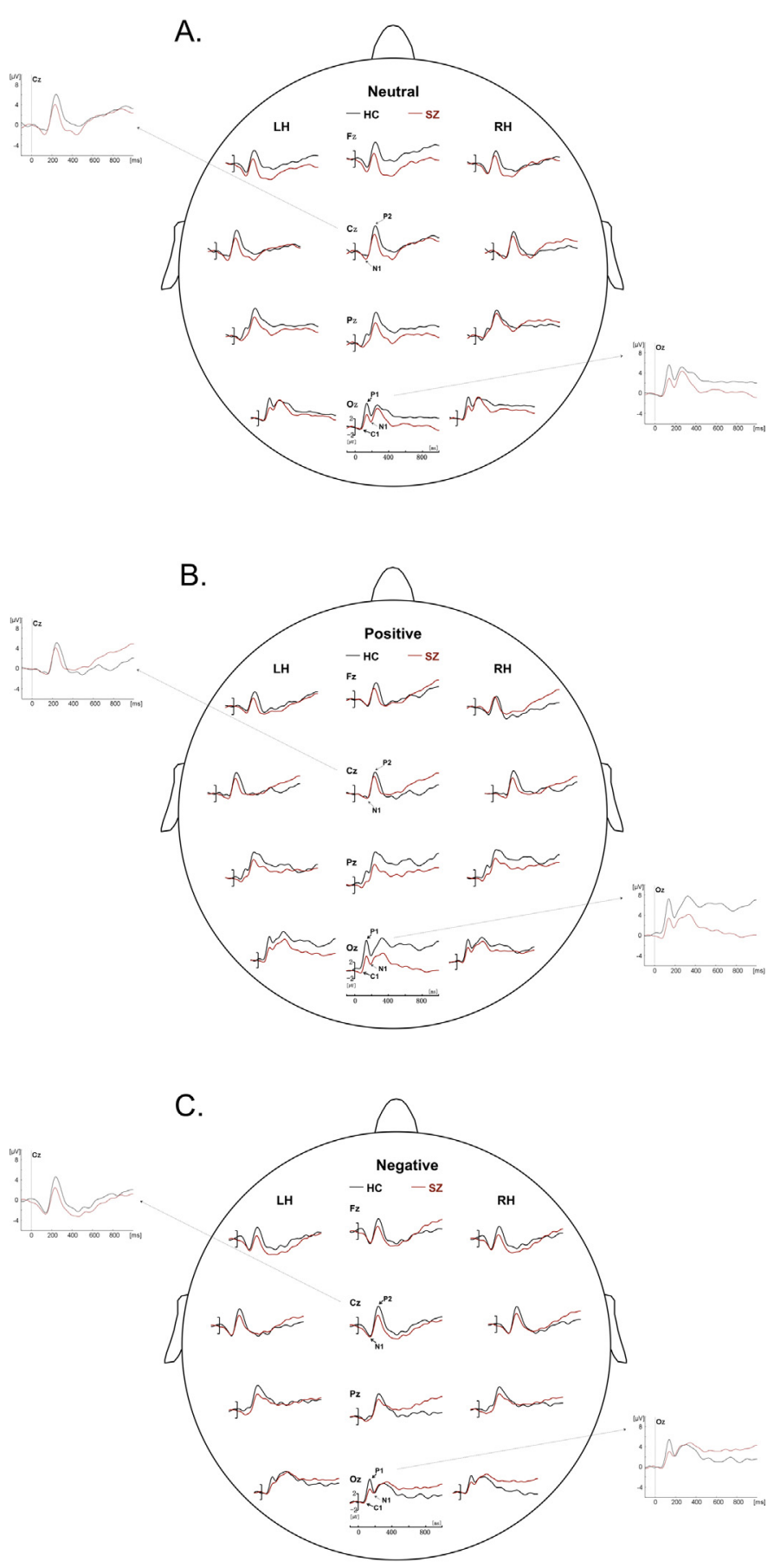

Fig. 1. Grand average waveforms illustrating group comparisons for neutral mood condition (Panel A), positive mood condition (Panel B), and negative mood condition (Panel C). Notes: $\mathrm{LH}=$ left hemisphere; $\mathrm{RH}=$ right hemisphere; $\mathrm{HC}=$ healthy controls; $\mathrm{SZ}=$ schizophrenia patients.

\subsection{Statistical analyses}

\subsubsection{ERPS}

Early sensory components localized to posterior and anterior sites, and broadly distributed later components were analyzed (see Fig. 1). For each ERP component, the midline MANOVA included mood (positive, negative, and neutral), and electrode (posterior components: $\mathrm{Pz}, \mathrm{POz}, \mathrm{Oz}$; anterior components: $\mathrm{Fz}, \mathrm{FCz}, \mathrm{Cz}$; for $\mathrm{P} 2$ and LPP: $\mathrm{Fz}, \mathrm{FCz}, \mathrm{Cz}, \mathrm{Pz}, \mathrm{POz}, \mathrm{Oz}$ ) as within-subjects factors, and group (schizophrenia, $\mathrm{HC}$ ) as between-subjects factor. For broadly distributed components, we have additionally included 'region' as within-subject factor (anterior: $\mathrm{Fz}, \mathrm{FCz}, \mathrm{Cz}$; posterior: $\mathrm{Pz}, \mathrm{POz}, \mathrm{Oz}$ ). Main effects and interactions were followed with pairwise comparisons with Bonferroni correction.

\subsubsection{Behavioral data}

A MANOVA with time (pre vs. post mood induction), mood type (neutral, positive, negative) and POMS scales as within-subject factors and group as between-subjects factor was computed to examine the effectiveness of mood induction procedure and to test for group differences. To test for group differences in ratings of pictures' valence and arousal, independent samples $t$-tests were computed.

\subsubsection{Correlational analyses}

Spearman's Rho correlations were performed in an exploratory analysis of the relationship between ERP amplitude (Pz for posterior components; $\mathrm{Cz}$ for anterior N1; and Cz, Pz, and Oz for broadly distributed P2 and LPP) and PANSS subscales scores, SANS anhedonia subscale scores, POMS self-report data (after mood elicitation), and IAPS pictures ratings. Additionally, in order to test for the effects of medication and disease chronicity, we correlated ERP amplitude (Pz for posterior components; Cz for anterior N1; Cz, Pz and $\mathrm{Oz}$ for and broadly distributed P2 and LPP) with mean equivalent chlorpromazine dosage, as well as with illness duration.

\section{Results}

\subsection{ERP results}

\subsubsection{Early sensory components}

3.1.1.1. Posterior components (C1, P1, N1). C1 - A main effect of group $(F(1,33)=4.549, p=0.04)$ revealed more negative $C 1$ amplitude in the schizophrenia group relative to $\mathrm{HC}$.

$P 1$ - A main effect of $\operatorname{mood}(F(2,32)=5.901, p<0.01)$ was observed: planned comparisons indicated more positive P1 for the positive mood condition relative to the negative mood condition. Importantly, a main effect of group $(F(1,33)=7.72, p<0.01)$ indicated that $\mathrm{P} 1$ was less positive in schizophrenia patients relative to HC.

$N 1$ - A main effect of group $(F(1,33)=4.027, p=0.053)$ revealed more negative-going $\mathrm{N} 1$ in schizophrenia than in HC.

3.1.1.2. Anterior component (N1). N1 - A significant effect of mood was observed for the anterior $\mathrm{N} 1(F(2,32)=3.587, p=0.039)$ : $\mathrm{N} 1$ was more negative in the negative relative to positive mood condition.

\subsubsection{Mid-latency components}

3.1.2.1. Broadly distributed component (P2). P2 - The significant main effect of $\operatorname{mood}(F(2,32)=4.409, p=0.02)$ showed more positive P2 amplitude for the positive relative to negative mood condition. Importantly, P2 was less positive in the schizophrenia group relative to $\mathrm{HC}(F(1,33)=5.956, p=0.02)$.

\subsubsection{Late components}

3.1.3.1. Broadly distributed component (LPP). LPP - No main effects or interactions were revealed by the midline analysis $(p>0.05)$.

Pre-and post mood induction self-report of mood (POMS): The main effect of time indicated a difference in mood ratings before and after the mood elicitation procedure $(F(1$, $33)=13.682, p<0.01)$. A significant main effect of mood type $(F(2,32)=4.007, p=0.028)$ revealed differences in mood ratings according to the type of mood elicitation. Of note, a significant time $\mathrm{x}$ mood type $\mathrm{x}$ POMS scale demonstrated specific differences in mood ratings $(F(14,20)=3.140, p=0.010)$. Pairwise comparisons showed that after neutral mood elicitation, reduced anger-hostility $(p=0.045)$ and reduced depression-dejection 
$(p=0.034)$ were reported; after positive mood elicitation, reduced depression-dejection $(p<0.01)$ was reported; after negative mood elicitation, reduced vigor-activity $(p=0.016)$, reduced friendliness $(p=0.032)$, increased confusion-bewilderment $(p<0.01)$, and reduced happiness $(p=0.012)$ were reported in both groups.

Importantly, a significant group $\times$ POMS scale interaction was found $(F(7,27)=5.897, p<0.001)$ : when compared with $\mathrm{HC}$, patients showed more depression-dejection $(p=0.017)$, more fatigue-inertia $(p=0.014)$, less vigor-activity $(p=0.014)$, less friendliness $(p<0.01)$, more confusion-bewilderment $(p<0.001)$, and less happiness $(p<0.01)$. Also, they tended to report more anger-hostility than HC $(p=0.056)$.

Correlations between ERP amplitude and POMS data: No significant correlations were found between any component and self-report mood scores in HC. However, self-reported negative mood in schizophrenia was negatively correlated with ERP amplitude of posterior C1 (correlation with depressiondejection ratings: rho $=-0.528, p=0.036$ ) and $\mathrm{N} 1$ (correlation with confusion-bewilderment ratings: $r h o=-0.8, p<0.001$ ), and of broadly-distributed P2 (correlation with depression-dejection ratings: $r h o=-0.541, p=0.031$; correlation with tension-anxiety ratings: $r h o=-0.637, p<0.01)$ to negative pictures only: the more positive the ERP amplitude, the lower the self-reported negative mood.

No significant ERP correlations were found with either PANSS or SANS scores, mean equivalent chlorpromazine dosage, or illness duration in the patient group.

Assessment of stimuli's affective properties: Affective pictures were similarly rated by both groups $(p>0.05$ for all comparisons see Table 2).

\section{Discussion}

This study investigated the processing of evocative pictures in the context of a mood induction procedure, in chronic medicated schizophrenia patients and their HC. Electrophysiologically, groups differed in the amplitude of early sensory posterior C1, P1 and N1, and anterior P2, indicating that sensory processing of both neutral and emotional pictures was abnormal. The late LPP component related to sustained emotional processing of evocative pictures did not differ between the two groups. This finding provided support for a more generalized visually based deficit in evocative pictures processing [5]. Furthermore, the absence of a significant group by mood interaction for these ERP components suggests that visual emotional content did not contribute to greater difficulties in the processing of visual information beyond sensory visual impairments described above [17]. This finding contrasts with results in the processing of auditory emotional information where abnormal P200 was reported to emotional prosody only [22]. In both groups, mood modulated differentially posterior P1 and anterior N1 indexing early sensory processes, and broadly distributed P2 indexing early categorization processes. Behaviorally, patients reported a more negative mood regardless of mood induction type [23], in spite of the fact that mood induction was effective in both patients and HC.

The association observed between early ERP posterior components in the negative mood condition only and self-reported negative mood in the patient group (the more positive the amplitude, the lower the negative mood ratings) suggests a relationship between early sensory abnormalities and increased negative mood. This finding suggests a specific role of visual sensory abnormalities in subsequent negative mood increases or, alternatively, the impact of negative mood on early ERP abnormalities.

In spite of altered early visual processing, no group differences were observed in the LPP, the component thought to index sustained emotional processing, associated with memory formation, i.e., more cognitive processes [7]. The lack of group differences in the LPP amplitude, coexisting with abnormalities in early sensory ERP components suggests that, in spite of abnormal sensory processing of visual evocative stimuli, these later operations were preserved in schizophrenia patients in this task. A similar finding was reported by Horan et al. [16]; in contrast, Horan et al. study [17] - in which pictures of different valence were presented in the same session - found LPP abnormalities in schizophrenia patients. This discrepancy has interesting methodological implications pointing to the importance of a design in highlighting different facets of pathology. The presence of female subjects in the Horan et al. [17] may have been another contributing factor to the pattern of observed results given differences in the way males and females respond to affective stimuli [12].

\subsection{Self-report of mood and ratings of affective properties of visual stimuli}

Even though schizophrenia patients reported affective changes consistent with the valence of the evocative pictures, they differed from $\mathrm{HC}$ in self-reported emotional experience. In general, patients reported more negative mood before and after the three types of mood induction relative to HC [23]. Given that no association was found between self-report of mood and negative or positive symptoms, it is likely that these ratings are independent from clinical symptoms.

No group differences were observed in the subjective valence and arousal ratings pointing to a dissociation between assessment of subjective feelings and ratings of the affective properties of visual stimuli in schizophrenia. This result suggests intact conscious emotion representation (as indexed by behavioral data), in spite of impaired automatic processing of visual information, as indexed by early ERP abnormalities. These results were likely not affected by medication as no significant correlations were found between ERP results and medication dose.

\subsection{Limitations}

Limitations of the study include a relatively small sample size and inclusion of male subjects only. Future studies should address these issues. The group differences in SES and educational level represent social downward drift and are part of the illness symptomatology. Therefore, adopting the approach used in most studies on schizophrenia, the groups were matched on parental SES believed to reflect better educational and socioeconomic opportunities that existed for schizophrenia subjects in their home of origin but were not realized as a consequence of the disease.

\section{Contributors}

APP and MN designed the study and wrote the protocol. APP and TL collected the data. APP, TL, MN, PGN, OFG and RWM managed the literature searches and analyses. APP, TL and MN undertook the statistical analysis and wrote the first draft of the manuscript. All authors contributed to and have approved the final manuscript.

\section{Acknowledgements}

This work was supported by a Post-Doctoral Grant (BPD/68967/2010) from Fundação para a Ciência e a Tecnologia-FCT (Portugal) and by a Research Grant (PTDC/PSI-PCL/116626/2010) funded by FCT and by European Regional Development Fund, both awarded to APP, and by two Grants from the National Institute of Mental Health - NIMH (RO1 MH 040799 grant awarded to RWM; RO3 MH 078036 grant awarded to MAN). 
We gratefully acknowledge all the participants of this study. We are also grateful to Elizabeth Thompson and Jenna Mezin for their help with data collection.

\section{References}

[1] N.C. Andreasen, Scale for Assessment of Negative Symptoms (SANS), University of Iowa, Iowa City, 1983.

[2] N.C. Andreasen, Scale for Assessment of Positive Symptoms (SAPS), University of Iowa, Iowa City, 1984.

[3] M.M. Bradley, P.J. Lang, Measuring emotion: the self-assessment manikin and the semantic differential, J. Behav. Ther. Exp. Psychiatry 25 (1994) 49-59.

[4] J. Brekke, D.D. Kay, K.S. Lee, M.F. Green, Biosocial pathways to functional outcome in schizophrenia, Schizophr. Res. 80 (2005) 213-225.

[5] P.D. Butler, I.Y. Abeles, N.G. Wiskopf, A. Tambini, M. Jalbrzikowski, M.E. Legatt, V. Zemon, J. Loughead, R.C. Gur, D.C. Javitt, Sensory contributions to impaired emotion processing in schizophrenia, Schizophr. Bull. (2009) 1095-1107.

[6] P.D. Butler, D.C. Javitt, Early-stage visual processing deficits in schizophrenia, Curr. Opin. Psychiatry 18 (2005) 151-157.

[7] F. Dolcos, R. Cabeza, Event-related potentials of emotional memory: encoding pleasant, unpleasant, and neutral pictures, Cogn. Affect. Behav. Neurosci. 2 (2002) 252-263.

[8] E.C. Dowd, D.M. Barch, Anhedonia and emotional experience in schizophrenia: neural and behavioral indicators, Biol. Psychiatry 67 (2010) 902-911.

[9] M.B. First, R.L. Spitzer, M. Gibbon, J.B.M. Williams, Structured Clinical Interview for DSM-IV Axis II Personality Disorders (SCID-II, version 2.0), Biometrics Research Department, New York State Psychiatric Institute, New York, 1995.

[10] M.B. First, R.L. Spitzer, M. Gibbon, J.B.M. Williams, Structured Clinical Interview for DSM-IV Axis I Diagnosis-Patient Edition (SCID-I/P, version 2.0), Biometric Research Department, New York State Psychiatric Institute, New York, 2002.

[11] A. Gerrards-Hesse, K. Spies, F.W. Hesse, Experimental inductions of emotional states and their effectiveness: a review, Br. J. Clin. Psychol. 85 (1994) 55-78.

[12] J.M. Goldstein, Sex, hormones and affective arousal circuitry dysfunction in schizophrenia, Horm. Behav. 50 (2006) 612-622.
[13] G. Gratton, M.G. Coles, E. Donchin, A new method for off-line removal of ocular artifact, Electroencephalogr. Clin. Neurophysiol. 55 (1983) 468-484.

[14] J.D. Henry, M.J. Green, A. de Lucia, C. Restuccia, S. McDonald, M. O‘Donnell, Emotion dysregulation in schizophrenia: reduced amplification of emotional expression is associated with emotional blunting, Schizophr. Res. 95 (2007) 197-204.

[15] A. Hollingshead, Four Factor Index of Social Status, Yale University Social Sciences Library, New Haven, 1976.

[16] W.P. Horan, D. Foti, G. Hajcak, J.K. Wynn, M.F. Green, Intact motivated attention in schizophrenia: evidence from event-related potentials, Schizophr. Res. 135 (2012) 95-99.

[17] W.P. Horan, J.K. Wynn, A.M. Kring, R.F. Simons, M.F. Green, Electrophysiologica correlates of emotional responding in schizophrenia, J. Abnorm. Psychol. 119 (2010) 18-30.

[18] S.R. Kay, A. Fiszbein, L.A. Opler, The positive and negative syndrome scale (PANSS) for schizophrenia, Schizophr. Bull. 13 (1987) 261-276.

[19] P.J. Lang, M.M. Bradley, B.N. Cuthbert, International affective picture system (IAPS): affective ratings of pictures and instruction manual. Technical Report A-8, University of Florida, Gainesville, FL, 2008.

[20] D.M. McNair, M. Lorr, L.F. Droppleman, Profile of Mood States, Educational \& Industrial Testing Service, San Diego, 1971/1981.

[21] R.C. Oldfield, The assessment and analysis of handedness: the Edinburgh Inventory, Neuropsychologia 9 (1971) 97-113.

[22] A.P. Pinheiro, E. Del Re, J. Mezin, P.G. Nestor, A. Rauber, R.W. McCarley, O.F. Gonçalves, M. Niznikiewicz, Sensory-based and higher-order operations contribute to abnormal emotional prosody processing in schizophrenia: an electrophysiological investigation, Psychol. Med. 43 (2013) 603-618.

[23] F. Schneider, R.C. Gur, R.E. Gur, D.L. Shtasel, Emotional processing in schizophrenia: neurobehavioral probes in relation to psychopathology, Schizophr. Res. 17 (1995) 67-75.

[24] M.J. Sergi, M.F. Green, Social perception and early visual processing in schizophrenia, Schizophr. Res. 59 (2002) 233-241.

[25] D. Wechsler, Wechsler Adult Intelligence Scale: Administration and Scoring Manual, third ed., The Psychological Corporation, San Antonio, 1997. 